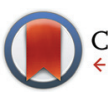

CrossMark \&lick for updates

Cite this: Polym. Chem., 2015, 6 , 7171

Received 29th July 2015

Accepted 25th August 2015

DOI: 10.1039/c5py01194a

www.rsc.org/polymers

\title{
Hypercrosslinked porous polymer nanosheets: 2D RAFT agent directed emulsion polymerization for multifunctional applications $\uparrow$
}

\author{
Wuxue Zhao, t $^{\mathrm{a}}$ Zongsheng Hou, ${ }^{\mathrm{a}}$ Zhaoquan Yao, ${ }^{\mathrm{a}}$ Xiaodong Zhuang, ${ }^{\text {a }}$ \\ Fan Zhang ${ }^{\star a}$ and Xinliang Feng ${ }^{a, b}$
}

\begin{abstract}
Two-dimensional hyper-cross-linked microporous polymers (2D HCPs) have been readily synthesized by emulsion polymerization using trithiocarbonate functionalized graphene oxide as a 2D reversible addition-fragmentation chain transfer (RAFT) agent. After a gel-type and permanently porous poly(vinylbenzyl chloride-co-divinylbenzene) precursor resin was grafted to the graphene surface, an $\mathrm{FeCl}_{3}$-promoted Friedel-Crafts reaction was carried out for benzyl chloride groups to construct a porous structure confined within 2D nanosheets. The resulting HCPs exhibited superhydrophobic behavior, and had a predominance of micropores with a specific surface area of up to $1224 \mathrm{~m}^{2} \mathrm{~g}^{-1}$. Moreover, they showed improved thermal stability in comparison with unadorned HCPs obtained without using the graphene template. Their $\mathrm{H}_{2}$ and $\mathrm{CO}_{2}$ capacities at 77 and $273 \mathrm{~K}$ reached 1.27 and $9.74 \mathrm{wt} \%$, respectively, at a relative pressure of 0.99. In addition, the as-synthesized 2D HCPs were used as carbon precursors to generate $2 \mathrm{D}$ porous carbons with a high specific surface area $\left(871 \mathrm{~m}^{2} \mathrm{~g}^{-1}\right)$ and high-performance electrochemical energy storage $\left(144 \mathrm{Fg}^{-1}\right.$ at $\left.0.5 \mathrm{~A} \mathrm{~g}^{-1}\right)$.
\end{abstract}

\section{Introduction}

Porous polymers ${ }^{1-6}$ with organic porous structures have attracted tremendous attention owing to their controllable porous features associated with prominent physical properties and potential applications, such as in light harvesting, ${ }^{7}$ sensing, ${ }^{8}$ gas separation ${ }^{9}$ and storage,${ }^{10}$ catalysis, ${ }^{11-13}$ and energy storage and conversion. ${ }^{14}$ Among them, hyper-crosslinked porous polymers (HCPs) represent one of the fastest developing types of porous polymers-not only because of their high specific surface areas and gas storage properties but also due to the easy availability of many controllable polymerization methods and the wide variety of functional monomers. ${ }^{15}$ Most of the reported HCPs have been amorphous, and were synthesized by traditional radical ${ }^{10,16-18}$ and oxidation ${ }^{19}$ polymerization methods, followed by an additional step for

\footnotetext{
${ }^{a}$ Shanghai Key Lab of Electrical Insulation and Thermal Ageing \& Shanghai Electrochemical Energy Devices Research Center, School of Chemistry and Chemical Engineering, Shanghai Jiao Tong University, Shanghai 200240, China. E-mail: zhuang@sjtu.edu.cn,fan-zhang@sjtu.edu.cn

${ }^{b}$ Center for Advancing Electronics Dresden \& Department of Chemistry and Food Chemistry, Technische Universitaet Dresden, 01062 Dresden, Germany

$\dagger$ †lectronic supplementary information (ESI) available. See DOI: 10.1039/ c5py01194a

$\$$ These two authors contributed equally to this work.
}

further cross-linking. Most of the previous studies on HCPs have focused on pore construction, tuning the gas ${ }^{19-21}$ or small-molecule ${ }^{22,23}$ separation/storage properties through optimizing the reaction conditions such as the monomer ratio, catalyst percentages, the temperature, and the reaction time. ${ }^{10,24}$ However, effort dedicated to morphology control has rarely been reported for HCPs. Only quasi-zero-dimensional microsphere $^{17,25}$ and three-dimensional monolith ${ }^{22,23}$ HCP types have been successfully synthesized. Two-dimensional (2D) HCPs are still under exploration owing to the lack of synthetic methods or $2 \mathrm{D}$ templates and the thermodynamic instability of 2D porous polymers in comparison with their zero- and three-dimensional porous counterparts.

Graphene, which consists of a honeycomb-like hexagonal array of $\mathrm{sp}^{2}$-bonded carbon atoms, exhibits many remarkable properties such as superior electrical conductivity, a large surface area, excellent mechanical flexibility, and high thermal/chemical stability. ${ }^{26,27}$ Graphene oxide (GO), one of the most widely synthesized derivatives of graphene and made from graphite by a modified Hummers' method, is an ideal 2D template for the preparation of $2 \mathrm{D}$ porous polymers because GO can be easily functionalized with many functional groups and solution processing. ${ }^{28-33}$ It is well known that the properties of graphene-based nanocomposites ${ }^{34}$ are highly dependent on the good dispersibility and interfacial interactions of graphene. In order to produce a homogeneous graphene 
dispersion during the preparation procedure, many attempts have been made to attach small molecules and linear polymers to the graphene surface by means of covalent bonds and weak interactions. ${ }^{35}$ Several grafting routes have been explored using either GO or functionalized GO with good dispersibility in various organic solvents as templates for the preparation of graphene-based 2D brushes and "sheet-coil" polymers. ${ }^{36}$ Ruoff and co-workers have prepared GO-based macroinitiators by the functionalization of GO with $\alpha$-bromoisobutyryl bromide for controlled styrene grafting via atom transfer radical polymerization (ATRP). ${ }^{37,38}$ Almost all the reported studies have focused on linear polymers, such as polystyrene, ${ }^{39,40}$ poly( $N$-vinylcarbazole $),{ }^{41}$ polymethacrylamide, ${ }^{42,43}$ and poly( $N$-isopropylacrylamide),${ }^{44}$ grafting from or grafting to graphene surfaces. To the best of the authors' knowledge, the preparation of $2 \mathrm{D}$ porous polymers using the $2 \mathrm{D}$ chain-transfer agent (CTA) promoted reversible addition-fragmentation chain transfer (RAFT) polymerization method has not yet been reported.

In this work, a highly soluble trithiocarbonate-based CTA was used to functionalize GO to act as a 2D CTA as well as a 2D template. Then, poly(vinylbenzyl chloride-co-divinylbenzene) (PVD) grafted GO was prepared by RAFT emulsion polymerization based on the as-prepared 2D CTA. After further hyper-cross-linking by an $\mathrm{FeCl}_{3}$-promoted Friedel-Crafts reaction of the benzyl chloride groups, graphene-based sandwichtype HCPs with a 2D morphology (GHCPs) were successfully prepared on a large scale ( $100 \mathrm{~g}$ per synthesis). The resulting GHCPs exhibited superhydrophobic behavior (contact angle $>150^{\circ}$ ) and had high specific surface areas of up to $1224 \mathrm{~m}^{2}$ $\mathrm{g}^{-1}$. They also exhibited an enhanced $\mathrm{H}_{2}$ capacity at $77 \mathrm{~K}(1.27$ versus $0.85 \mathrm{wt} \%)$ and $\mathrm{CO}_{2}$ capacity at $273 \mathrm{~K}(9.74$ versus $8.80 \mathrm{wt}$ $\%$ ) at a relative pressure of 0.99 in comparison with unadorned HCP obtained without using the graphene template. The high specific surface areas and the enhanced $\mathrm{H}_{2}$ and $\mathrm{CO}_{2}$ capacities can be the contributions of the $2 \mathrm{D}$ graphene template. Owing to the carbon backbone of graphene, GHCPs were used to prepare porous carbon nanosheets without removing the template. The as-prepared porous carbon nanosheets exhibited the typical 2D morphology and a high surface area $\left(871 \mathrm{~m}^{2}\right.$ $\left.\mathrm{g}^{-1}\right)$, and were successfully used in electrochemical capacitors $\left(144 \mathrm{~F} \mathrm{~g}^{-1}\right.$ at $\left.0.5 \mathrm{~A} \mathrm{~g}^{-1}\right)$. These results indicate that this method is a good approach for the preparation of 2D HCPs and 2D porous carbons with multifunctional applications.

\section{Experimental section}

\section{Reagents and chemicals}

Natural flake graphite, 1-dodecanethiol, carbon disulfide, aliquot 336, 1,3-diaminopropane, $N$-hydroxy-succinimide (NHS), 1-(3-dimethylaminopropyl)-3-ethylcarbodiimide hydrochloride (EDC.HCl), divinylbenzene (DVB), 2,2'-azobis(2methylpropio-nitrile) (AIBN), and poly(vinyl alcohol) (PVA) were purchased from Aladdin (Shanghai). Vinylbenzyl chloride (VBC) was purchased from TCI. 1,2-Dichloroethane (DCE), tetrahydrofuran (THF), $\mathrm{FeCl}_{3}$, and other chemicals were purchased from Sinopharm Chemical Reagent Co. (Shanghai). THF, DCE and triethylamine were dried by standard methods before use.

\section{Functionalization of graphene oxide with amine groups ${ }^{45}$}

GO was prepared from natural graphite flakes by a modified Hummers' method. ${ }^{46}$ GO sheets $(1.0 \mathrm{~g})$ were added to dry DMF (250 mL). After ultrasonication for $24 \mathrm{~h}$, NHS $(3.42 \mathrm{~g}$, $30 \mathrm{mmol})$ and EDC. $\mathrm{HCl}(5.75 \mathrm{~g}, 3.2 \mathrm{mmol})$ were added to GO dispersion at $0{ }^{\circ} \mathrm{C}$. After stirring for $2 \mathrm{~h}, 1,3$-diaminopropane $(5.0 \mathrm{~mL})$ was added, and the solution was stirred overnight at room temperature. After filtration and washing with water and ethanol, the resulting powder was freeze-dried and denoted as GO- $\mathrm{NH}_{2}$.

\section{Preparation of 2D CTA}

2-(Dodecylthiocarbonothioylthio)-2-methylpropionic acid (DDAT), which is a widely used and highly soluble chain transfer agent (CTA) for RAFT polymerization, was synthesized according to reported methods. ${ }^{47,48}$ GO-NH to dry DMF (250 mL) and sonicated for a few hours. Then, triethylamine $(5.0 \mathrm{~mL})$ was added to the dispersion. Acylchloride-DDAT was prepared as follows: DDAT $(5.0 \mathrm{~g})$ and excess thionyl chloride $(20 \mathrm{~mL})$ were added to a $50 \mathrm{~mL}$ three-neck round-bottom flask and refluxed overnight under a nitrogen atmosphere. Then, unreacted thionyl chloride was completely removed under reduced pressure and washed with anhydrous THF $(8 \mathrm{~mL} \times 3)$. Lastly, the acylchloride-DDAT in THF $(10 \mathrm{~mL})$ was transferred to the above $\mathrm{GO}-\mathrm{NH}_{2}$ dispersion very slowly under vigorous stirring in an ice bath. The mixtures were stirred at room temperature for $24 \mathrm{~h}$. The resulting DDAT functionalized GO (GO-DDAT) was washed with $\mathrm{CH}_{2} \mathrm{Cl}_{2}$ and ethanol, and dried overnight under vacuum at $50{ }^{\circ} \mathrm{C}$.

\section{RAFT emulsion polymerization}

VBC/DVB modified GO precursor resin was prepared by conventional RAFT emulsion polymerization. ${ }^{49,50}$ The aqueous phase consisted of distilled water $(50 \mathrm{~mL})$, PVA $(0.38 \mathrm{~g})$ and $\mathrm{NaCl}(0.17 \mathrm{~g})$. The organic phase comprised GO-DDAT (100 mg), VBC (6.37 g, $41.7 \mathrm{mmol})$, DVB (0.16 g, $1.2 \mathrm{mmol})$, and AIBN (33 mg). The organic phase was suspended in the aqueous phase at $80{ }^{\circ} \mathrm{C}$ by a stirring speed of $425 \mathrm{rpm}$ under a $\mathrm{N}_{2}$ atmosphere. After $8 \mathrm{~h}$, the beads were filtered and washed three times with water, methanol and diethyl ether. After drying under vacuum overnight at $60{ }^{\circ} \mathrm{C}$, the copolymer grafted GO (GO-PVD) was produced. Bare PVD without using a graphene template was also synthesized using the same method.

\section{Synthesis of 2D hypercrosslinked microporous polymer}

The VBC/DVB modified GO precursor resin beads (3.25 g) were swollen in DCE $(40 \mathrm{~mL})$ for $3 \mathrm{~h}$. The mixture was cooled in an ice bath before adding $\mathrm{FeCl}_{3}(2.0 \mathrm{~g})$. After achieving a uniform dispersion of $\mathrm{FeCl}_{3}$ throughout the precursor beads in $2 \mathrm{~h}$, the mixture was heated to $80{ }^{\circ} \mathrm{C}$ for $12 \mathrm{~h}$. After the resulting hyper- 
crosslinked beads were filtered, the catalyst was washed out using methanol, a mixture of acetone and $\mathrm{HCl}(0.5 \mathrm{M})$, and deionized water. After drying under vacuum at $60{ }^{\circ} \mathrm{C}$, the resulting 2D hypercrosslinked microporous polymer (2D-HCP) was produced. In the control experiment, GHCP-1, GHCP-2 and GHCP-3, denoting $100 \mathrm{mg}, 200 \mathrm{mg}$ and $800 \mathrm{mg}$ of GO-DDAT, were used in above procedure respectively. According to the same procedure, bare PVD was also used to prepare HCP in order to compare with GHCPs.

\section{Characterization}

FT-IR spectra, using $\mathrm{KBr}$ tablets containing the sample, were recorded on a Spectrum 100 (PerkinElmer, Inc., USA) spectrometer with a scan range of $4000-400 \mathrm{~cm}^{-1}$. The thermal degradation was analyzed by means of thermogravimetric analysis (TGA), which was measured by using a Q5000IR (TA Instruments, USA) thermogravimetric analyzer with a heating rate of $20{ }^{\circ} \mathrm{C} \mathrm{min}^{-1}$ under nitrogen flow. Scanning electron microscopy (SEM) measurements were performed on a FEI Sirion-200 (FEI Co., USA) field emission scanning electron microscope. Transmission electron microscopy (TEM) images were taken with an EOL-2100 (JEOL Ltd, Japan) electron microscope at an operating voltage of $200 \mathrm{kV}$. The suspensions of the samples were dropped onto a copper grid covered with lacey support films. The nitrogen content was determined with a Vario ELIII/Isoprime (Elementar Co., Germany) isotope ratio mass spectrometer. The Brunauer-Emmett-Teller (BET) specific areas were measured on an ASAP $2010 \mathrm{M}+\mathrm{C}$ (Micromeritics Inc., USA) surface area and porosimetry analyzer based on $\mathrm{N}_{2}$ adsorption. The hydrogen and carbon dioxide sorptions were measured with Autosorb-iQA3200-4 adsorption equipment (Quantatech Co., USA).

Electrochemical capacity characterization was conducted on an EG \& potentiostat/galvanostat Model 2273 advanced electrochemical system. A conventional cell with a three-electrode configuration was employed throughout this study. The working electrode was prepared by mixing GHCP-1-900 with carbon black (Mitsubishi Chemicals, Inc.) and a polytetrafluoroethylene (PTFE) binder. The weight ratio of GHCP-1900, carbon black and PTFE was $80: 10: 10$. A platinum foil was applied as a counter electrode with a $\mathrm{Hg} / \mathrm{HgO}$ electrode as a reference electrode. The experiments were carried out in $6 \mathrm{M}$ $\mathrm{KOH}$ solution. The potential range was between -1 and $0 \mathrm{~V}$ $(\mathrm{Ag} / \mathrm{AgCl})$ at different scan rates at ambient temperature.

\section{Results and discussion}

The strategy for the synthesis of GHCPs is presented in Scheme 1. First, a GO sheet was functionalized by 1,3-diaminopropane in the presence of NHS and EDC $\cdot \mathrm{HCl}$ and then by acylchloride-DDAT in the presence of $\mathrm{Et}_{3} \mathrm{~N}$. The sulfur content of the produced DDAT-modified GO (DDAT) reached $2.3 \mathrm{wt} \%$. Using GO-DDAT as a 2D CTA, RAFT emulsion polymerization was carried out on graphene surfaces using 1,4-divinylbenzene and 4-vinylbenzyl chloride as monomers in water along with

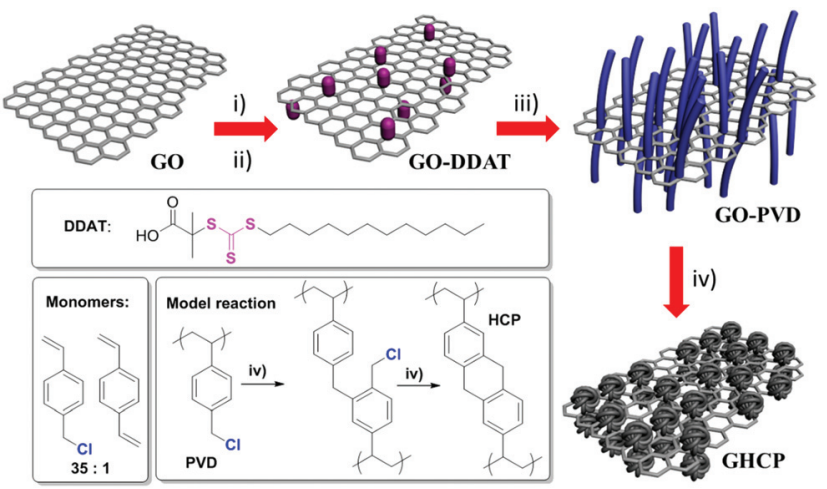

Scheme 1 Preparation of GHCPs: (i) N-hydroxy-succinimide (NHS), 1-ethyl-3-(3-dimethylaminopropyl)carbodiimide hydrochloride (EDC. $\mathrm{HCl}$ ), 1,3-diaminopropane, water, $0{ }^{\circ} \mathrm{C}, 12 \mathrm{~h}$; (ii) $\mathrm{N}_{2}$, acyl chloride$S$-1-dodecyl- $S^{\prime}-\left(\alpha, \alpha^{\prime}\right.$-dimethyl- $\alpha^{\prime \prime}$-acetic acid)trithiocarbonate (DDAT), dry dimethylformamide, $\mathrm{Et}_{3} \mathrm{~N}, 0^{\circ} \mathrm{C}, 24 \mathrm{~h}$; (iii) $\mathrm{N}_{2}$, water, polyvinyl alcohol (PVA), $\mathrm{NaCl}$, AIBN, 1,4-divinylbenzene, 4-vinylbenzyl chloride, $80^{\circ} \mathrm{C}, 8 \mathrm{~h}$; (iv) 1,2-dichloroethane, $\mathrm{FeCl}_{3}, 80^{\circ} \mathrm{C}, 12 \mathrm{~h}$.

AIBN, PVA, and NaCl. Afterwards, the copolymer-modified GO (GO-PVD) that was produced was hyper-cross-linked by using an $\mathrm{FeCl}_{3}$-catalyzed Friedel-Crafts reaction. Finally, 2D sandwich-type GHCPs were obtained by vacuum drying. In this procedure, GO-DDAT not only showed very good dispersibility in most organic solvents but acted as a $2 \mathrm{D}$ template for polymerization on graphene surfaces. In a control experiment, GHCP-1, GHCP-2, and GHCP-3 (denoting 1, 2, and 3 equivalents of GO-DDAT (see the Experimental section)) were obtained using the same procedure. The color of HCP, GHCP-1, GHCP-2, and GHCP-3 darkens as the GO-DDAT content increases (Fig. 1a). Moreover, the uniform color of the GHCPs indicates that the porous polymer has grafted uniformly to the GO flakes. The unadorned hyper-cross-linked porous polymer was also prepared without using the graphene template for comparison in this work (denoted HCP).

The morphology and microstructure of the as-prepared GHCPs were investigated by scanning electron microscopy (SEM) and transmission electron microscopy (TEM). All of the GHCPs showed similar sheet morphologies (Fig. 1 and S1 $\dagger$ ). Thus, the results of GHCP-3 are discussed here as a typical example. As shown in Fig. 1b, many free-standing sheets with a morphology similar to that of graphene and with sizes ranging from $500 \mathrm{~nm}$ to several micrometers were observed. In addition, these porous polymer sheets exhibited wrinkles and flexible features that stand in contrast to the rigid inorganic porous silica sheets reported previously. ${ }^{51}$ No free porous polymer particles or naked graphene sheets were observed in either the TEM or SEM investigations. This suggests that, as expected, most of the monomers have been polymerized on the surface of graphene. The control sample (HCP) without the GO-DDAT template exhibited the amorphous nanoparticle structure reported previously (Fig. S2 $\dagger$ ). ${ }^{52}$ These results strongly suggest the crucial role of graphene as a substrate in the grafting of HCPs in a 2D manner. ${ }^{53}$ 


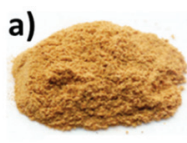
HCP

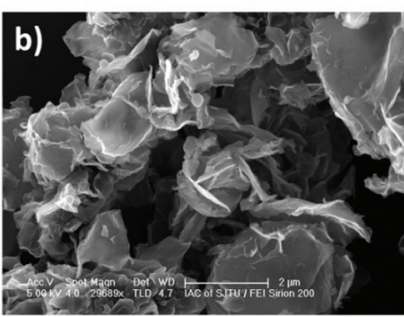

d)

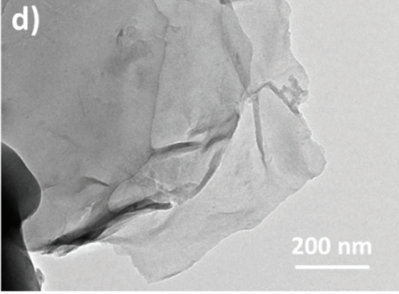

Fig. 1 (a) HCP and the GHCPs. (b, c) SEM and (d, e) TEM images of GHCP-3.

To analyze the molecular structure of the HCPs, Fourier transform infrared (FT-IR) spectroscopy was employed. Typical FT-IR spectra for GO, GO-DDAT, unadorned HCP, and GHCP-3 are shown in Fig. 2. The broad band at $3430 \mathrm{~cm}^{-1}$ is attributed to $\mathrm{O}-\mathrm{H}$ stretching vibrations, ${ }^{54}$ which might originate from carboxylic acid and hydroxyl groups on the GO sheets or from adsorbed water. GO shows two adjacent peaks at 1720 and $1645 \mathrm{~cm}^{-1}$, which correspond to $\mathrm{C}=\mathrm{O}$ and $\mathrm{C}=\mathrm{C}$ stretching vibrations. ${ }^{55}$ The peak at $1065 \mathrm{~cm}^{-1}$ represents $\mathrm{C}-\mathrm{O}$ stretching vibrations, ${ }^{56}$ which indicate the presence of the epoxide group in GO. ${ }^{57}$ In Fig. 2, unadorned HCP and GHCP-3 show a significant peak at $1610 \mathrm{~cm}^{-1}$ (skeletal vibrations from graphitic domains), attributable to aromatic $\mathrm{C}=\mathrm{C} .{ }^{54}$ Additionally, the

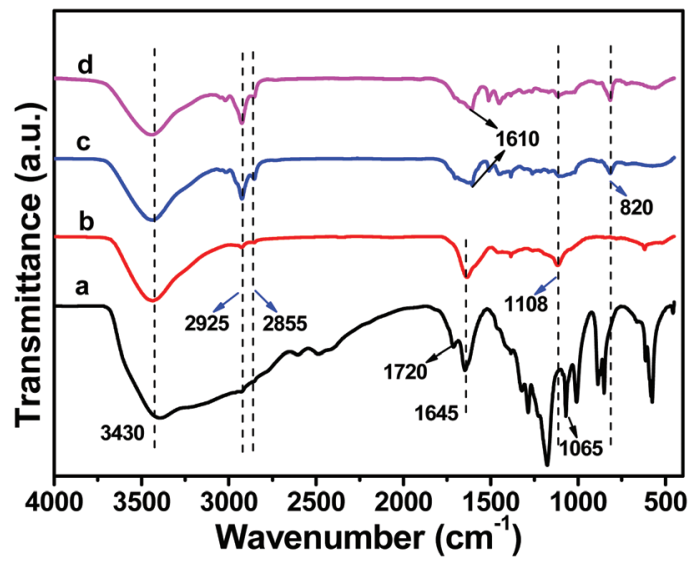

Fig. 2 FT-IR spectra of (a) GO, (b) GO-DDAT, (c) unadorned HCP and (d) GHCP-3. peaks at 2925 and $2855 \mathrm{~cm}^{-1}$ assigned to the methylene stretch indicate the existence of $\mathrm{CH}_{2}$ or $\mathrm{CH}$ groups. ${ }^{54}$ In particular, the peak at $1065 \mathrm{~cm}^{-1}$ for GO shifted to $1108 \mathrm{~cm}^{-1}$ for GO-DDAT and GHCP-3, which might be attributable to hydrogen bond interactions. ${ }^{58}$ Compared with GO and GO-DDAT, unadorned HCP and GHCP-3 have a distinctive peak at $820 \mathrm{~cm}^{-1}$, corresponding to $\mathrm{C}-\mathrm{Cl}$ stretching, ${ }^{59}$ which suggests the existence of a few unreacted $\mathrm{Ar}-\mathrm{CH}_{2}-\mathrm{Cl}$ groups (see the model reaction in Scheme 1).

In order to reveal the porous structures of the 2D HCPs, the $\mathrm{N}_{2}$ adsorption/desorption properties of GHCPs and unadorned HCP were studied (Fig. 3 and Table 1). All the samples showed a distinct step in the $\mathrm{N}_{2}$ adsorption isotherm at a low relative pressure $\left(P / P_{0}<0.1\right)$, which corresponds to gas sorption in micropores. ${ }^{60}$ According to the IUPAC classification, ${ }^{61}$ these samples give rise to type I nitrogen gas sorption isotherms with $\mathrm{H} 3$ hysteresis loops, indicating that the materials contain micro- and mesopores. The specific surface areas of 1224 and $1693 \mathrm{~m}^{2} \mathrm{~g}^{-1}$ (the former calculated from the BrunauerEmmett-Teller (BET) theory, the latter from Langmuir's theory) for GHCP-1 are larger than those of HCP (600 and $\left.832 \mathrm{~m}^{2} \mathrm{~g}^{-1}\right)$, respectively. This result could be attributable to the graphene template and the resulting 2D morphology. This analysis was further confirmed by a pore size study. Pore size distributions calculated by the nonlocal density functional theory (NL-DFT) method for HCP and the GHCPs are illustrated in Fig. 3b. All of the samples exhibited pore diameters centering around $1.6 \mathrm{~nm}$, implying that they contained mainly micropores $(<2.0 \mathrm{~nm})$. The GHCPs also showed several broad peaks between 2.3 and $6.0 \mathrm{~nm}$, implying the existence of a few mesopores (2.0-50.0 nm). These results-when considered together-indicate that the GHCPs prepared in this study are new hierarchical porous organic materials with a $2 \mathrm{D}$ morphology.
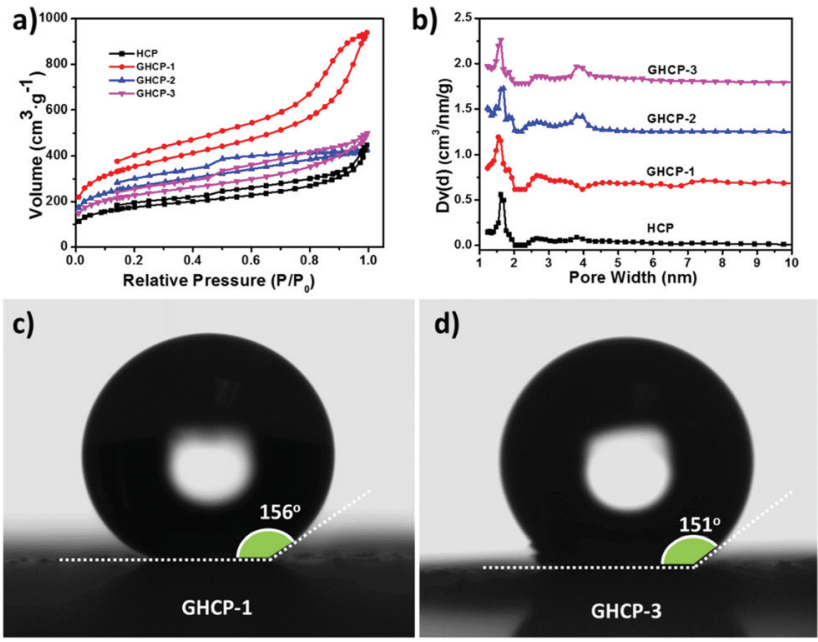

Fig. 3 (a) Nitrogen adsorption and desorption isotherms of unadorned HCP and the GHCPs at $77 \mathrm{~K}$. (b) The pore size distribution calculated by the NL-DFT method. (c, d) Illustrative images of a water droplet on the superhydrophobic surfaces. 
Table 1 Nitrogen physisorption properties and $\mathrm{H}_{2}$ and $\mathrm{CO}_{2}$ uptake for $\mathrm{HCP}$ and the GHCPs

\begin{tabular}{|c|c|c|c|c|c|c|c|}
\hline & \multirow{2}{*}{$\begin{array}{l}S_{\mathrm{BET}}{ }^{a} \\
\left(\mathrm{~m}^{2} \mathrm{~g}^{-1}\right)\end{array}$} & \multirow{2}{*}{$\begin{array}{l}S_{\text {Lang }}{ }^{a} \\
\left(\mathrm{~m}^{2} \mathrm{~g}^{-1}\right)\end{array}$} & \multirow{2}{*}{$\begin{array}{l}V_{\text {tot }}^{b} \\
\left(\mathrm{~cm}^{3} \mathrm{~g}^{-1}\right)\end{array}$} & \multicolumn{2}{|c|}{$\mathrm{H}_{2}$ uptake $^{c}$} & \multicolumn{2}{|c|}{$\mathrm{CO}_{2}$ uptake $^{c}$} \\
\hline & & & & $77 \mathrm{~K}$ & $87 \mathrm{~K}$ & $273 \mathrm{~K}$ & 2981 \\
\hline CP & 600 & 832 & 0.69 & 0.85 & 0.63 & 8.80 & 5.86 \\
\hline GHCP-1 & 1224 & 1693 & 1.45 & 1.27 & 0.82 & 9.74 & 6.19 \\
\hline GHCP-2 & 971 & 1344 & 0.71 & 1.16 & 0.77 & 9.84 & 6.22 \\
\hline GHCP-3 & 780 & 1083 & 0.78 & 1.09 & 0.67 & 9.06 & 5.82 \\
\hline
\end{tabular}

${ }^{a}$ Surface area calculated from the $\mathrm{N}_{2}$ adsorption isotherm using the BET method and the Langmuir method, respectively. ${ }^{b}$ Total pore volume at $P / P_{0}=0.99 .{ }^{c}$ Gravimetric gas uptake (wt\%) for $\mathrm{H}_{2}$ and $\mathrm{CO}_{2}$ at $P / P_{0}=0.99$.

Additionally, the hydrophobic property of the GHCPs was studied to better understand their unique hierarchical porous structures. First, ethanol dispersions of the GHCPs and HCP were sprayed onto glass surfaces. After drying in air at room temperature overnight, water droplets were placed on these surfaces (Fig. 3c and d). All the GHCPs showed a contact angle of $155 \pm 4^{\circ}$, which is typically superhydrophobic, and marginally larger than that of HCP $\left(151 \pm 1^{\circ}\right)$. The slightly improved contact angle may result from the $2 \mathrm{D}$ morphology, and could prove useful for the construction of better superhydrophobic materials.

Hydrogen is an ideal and attractive candidate for industrial applications, such as ammonia synthesis, automobile fuels, and metallurgy, because of its abundance, renewability, and environmentally "clean" aspect. ${ }^{62}$ Hydrogen isotherms for HCP and the GHCPs up to a maximum relative pressure $\left(P / P_{0}\right)$ of 0.99 at $77 \mathrm{~K}$ were examined, as shown in Fig. 4a. GHCP-1, GHCP-2, and GHCP-3 exhibited hydrogen capacities of 142, 130 , and $122 \mathrm{~cm}^{3} \mathrm{~g}^{-1}(1.27,1.16$, and $1.09 \mathrm{wt} \%)$, respectively,
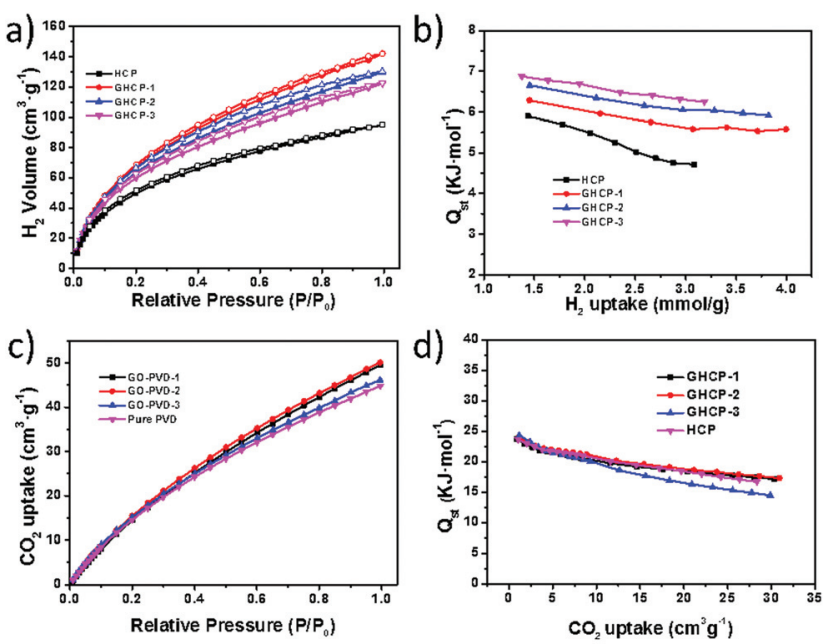

Fig. 4 Volumetric (a) $\mathrm{H}_{2}$ and (c) $\mathrm{CO}_{2}$ isotherms of unadorned $\mathrm{HCP}$ and the GHCPs at $77 \mathrm{~K}$ and $273 \mathrm{~K}$, respectively. Isosteric heat of adsorption of (b) $\mathrm{H}_{2}$ and (d) $\mathrm{CO}_{2}$ for $\mathrm{HCP}$ and the GHCPs at different gas loadings. which are all higher than that of HCP $\left(95 \mathrm{~cm}^{3} \mathrm{~g}^{-1}, 0.85 \mathrm{wt} \%\right)$. Obviously, the hydrogen capacities decrease along with the content of the graphene template. These results reveal that graphene contributes to the total hydrogen capacity, and underline the importance of a high graphene surface area for maximal hydrogen uptake capacity $\left(S_{\mathrm{BET}}\right.$ : GHCP-1 > GHCP-2 > GHCP-3). Based on a variant of the Clausius-Clapeyron equation, the hydrogen isosteric heat of adsorption can be calculated (Fig. 4b). ${ }^{63}$ At low coverage, GHCP-1, GHCP-2, and GHCP-3 exhibited the highest adsorption enthalpies at 6.3, 6.7, and $6.9 \mathrm{~kJ} \mathrm{~mol}^{-1}$, respectively (Fig. 4b), which are slightly larger than that of unadorned $\mathrm{HCP}\left(5.9 \mathrm{~kJ} \mathrm{~mol}^{-1}\right)$. A more rapid decrease to $4.7 \mathrm{~kJ} \mathrm{~mol}^{-1}$ for unadorned HCP was shown in the high hydrogen uptake region. The high heat of adsorption may stem from the narrower pores, which allow stronger overall interactions of the guest gas molecules because of additional interactions with the opposite walls. ${ }^{64}$ The adsorption enthalpies of $\mathrm{H}_{2}$ for the GHCPs increased with the increase in graphene content, indicating that the interaction between $\mathrm{H}_{2}$ and the sample was increasingly dominating that between $\mathrm{H}_{2}$ molecules because of the higher content of the graphene template.

In order to study the different gas capacities of the GHCPs, their $\mathrm{CO}_{2}$ storage capacities were investigated. The $\mathrm{CO}_{2}$ adsorption isotherms of HCP and the GHCPs at $273 \mathrm{~K}$ are presented in Fig. 4c. GHCP-1, GHCP-2, GHCP-3, and unadorned HCP exhibited $\mathrm{CO}_{2}$ uptake of 9.74, 9.84, 9.06, and $8.80 \mathrm{wt} \%$, respectively (see Table 1). It was found that adsorbed $\mathrm{CO}_{2}$ was not significantly affected in comparison with $\mathrm{H}_{2}$ when employing the graphene template, implying that the interactions between $\mathrm{CO}_{2}$ molecules and samples were unaffected by the graphene template. Unadorned HCP showed $\mathrm{CO}_{2}$ adsorptions of $44.8 \mathrm{~cm}^{3} \mathrm{~g}^{-1}(273 \mathrm{~K})$ and $29.8 \mathrm{~cm}^{3} \mathrm{~g}^{-1}(298 \mathrm{~K})$ at a relative pressure $\left(P / P_{0}\right)$ of 0.99 . Under the same pressure conditions, the $\mathrm{CO}_{2}$ adsorptions of GHCP-1 were 49.6 and $31.5 \mathrm{~cm}^{3} \mathrm{~g}^{-1}$ at 273 and $298 \mathrm{~K}$, respectively. The isosteric enthalpies $\left(Q_{\mathrm{st}}\right)$ for $\mathrm{CO}_{2}$ were also calculated, and are shown in Fig. $4 \mathrm{~d}$. The $\mathrm{CO}_{2}$ $Q_{\text {st }}$ value at lower coverage mainly reflects the interaction strength between $\mathrm{CO}_{2}$ and the sorbent. ${ }^{65}$ As shown in Fig. $4 \mathrm{~d}$, the $\mathrm{CO}_{2} Q_{\mathrm{st}}$ values of HCP and the GHCPs were found to be almost the same in the range $0-8.0 \mathrm{~cm}^{3} \mathrm{~g}^{-1}$, indicating that (1) the interactions between $\mathrm{CO}_{2}$ and the porous samples are weak and (2) morphology and the graphene template have a limited impact on $\mathrm{CO}_{2}$ uptake. ${ }^{66}$

The thermal behaviors of unadorned HCP and the GHCPs were recorded by thermal gravimetric analysis (TGA) at a heating rate of $20{ }^{\circ} \mathrm{C} \mathrm{min}^{-1}$ under a nitrogen atmosphere (Fig. 5a). Unadorned HCP is thermally unstable below $400{ }^{\circ} \mathrm{C}$. Remarkably, the weight loss for the GHCPs started as high as $300{ }^{\circ} \mathrm{C}$, and became rapid only in the range between 400 and $600{ }^{\circ} \mathrm{C}$, which might be attributed to the alkane structure and oxygen-containing groups in the materials. The improved thermal stability of the GHCPs may be attributable to the high thermal stability of the graphene sheets. ${ }^{40}$ After $600{ }^{\circ} \mathrm{C}$, the residual weight for all samples was almost constant, which suggests that the samples had been converted to stable carbon 

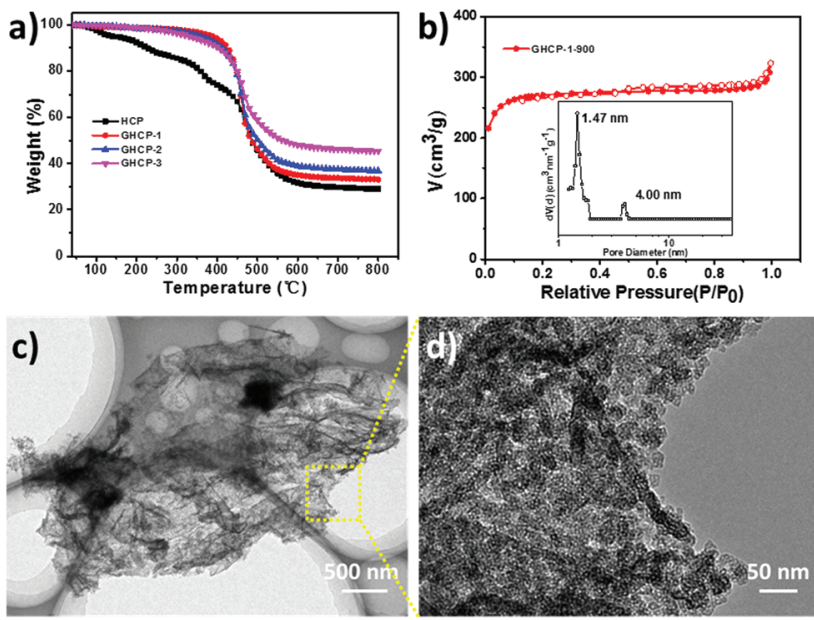

Fig. 5 (a) TGA curves of $\mathrm{HCP}$ and the GHCPs at a heating rate of $20^{\circ} \mathrm{C}$ $\mathrm{min}^{-1}$ under nitrogen flow. (b) Nitrogen adsorption and desorption isotherms at $77 \mathrm{~K}$ (inset: the pore size distribution calculated by the NL-DFT method). (c, d) TEM images of GHCP-1-900.

structures. At $800{ }^{\circ} \mathrm{C}$, the residual weights of HCP, GHCP-1, GHCP-2, and GHCP-3 were 28.9, 33.1, 36.8 and 45.4 wt\%, respectively, which also indicates that the graphene templates in the GHCPs enhance the thermal stability. The porous nature of GHCP-1-900 was further studied using nitrogen physisorption measurements. It was found that the isotherms for GHCP-1-900 were type I (Fig. 5b). The BET and Langmuir surface areas of GHCP-1-900 were calculated as 871 and $1212 \mathrm{~m}^{2} \mathrm{~g}^{-1}$, respectively. The pore size distribution of GHCP-1-900 is presented as an inset in Fig. 5b. The two peaks at 1.47 and $4.00 \mathrm{~nm}$ indicate that the hierarchical porous carbon nanosheets can be easily produced via the direct pyrolysis of $2 \mathrm{D}$ HCPs. These results also indicate that the high surface areas of 2D HCP-derived porous carbon nanosheets can be achieved by taking advantage of the $2 \mathrm{D}$ structure of graphene sheets. TEM images of GHCP-1-900 are shown in Fig. 5c and $d$ : free-standing sheets with a morphology similar to that of graphene and several micrometers in size can be observed. It is clear that the $2 \mathrm{D}$ morphology with visible wrinkles can be well preserved after high temperature pyrolysis of GHCP-1. Interestingly, alternating light and dark regions can be easily seen in the enlarged TEM image, indicating the possible creation of a porous carbon during pyrolysis.

The high specific surface area and unique 2D nanosheet morphology as well as the porous structure of these newly prepared porous carbon nanosheets hold promise for their application in electrochemical capacitors. Therefore, the electrochemical capacitance of GHCP-1-900 was examined under alkaline conditions ( $6 \mathrm{M} \mathrm{KOH})$ as a proof of concept. Typically, as shown in Fig. 6a, symmetric and horizontal cyclic voltammetry (CV) curves at a scan rate of $5-100 \mathrm{mV} \mathrm{s}^{-1}$ were observed for GHCP-1-900, indicating ideal capacitive behavior. The capacitive performance was further investigated in galvanostatic charge/discharge cycling experiments (Fig. 6b). On the a)
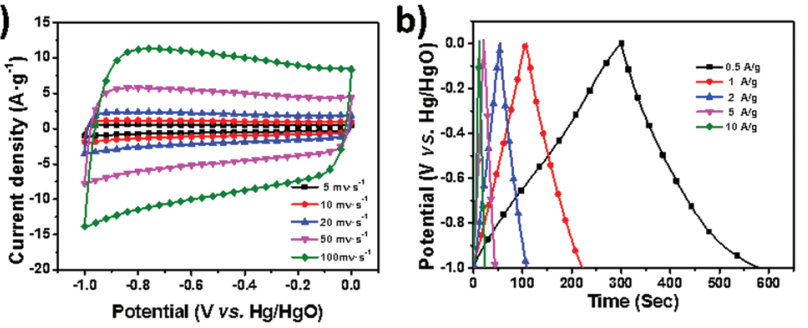

Fig. 6 (a) CV curves of GHCP-1-900 at 5-100 mV s${ }^{-1}$ in $6 \mathrm{M} \mathrm{KOH}$ aqueous solution. (b) Galvanostatic charge/discharge curves for GHCP-1-900 at a current density of $0.5-10 \mathrm{~A} \mathrm{~g}^{-1}$.

basis of the discharge curve, the specific capacitances of GHCP-1-900 were calculated to be 144, 116, 112, 127, and 122 $\mathrm{F} \mathrm{g}^{-1}$ at $0.5,1,2,5$, and $10 \mathrm{~A} \mathrm{~g}^{-1}$, respectively. For supercapacitor applications, the graphene layer in sandwich-type GHCP-1900 can act as both a mini-current collector and a long-distance in-plane charge transporter during the charge and discharge processes, by taking advantage of the high electrical conductivity and the 2D electron transport properties of graphene. Additionally, inter-/intra-layer electrolyte transport also contributes to the superior capacitance, given that the active surface of nanosheets can be efficiently exposed to the electrolyte. ${ }^{31}$

\section{Conclusions}

In summary, GHCPs based on vinylbenzyl chloride and divinylbenzene were successfully prepared by a RAFT emulsion polymerization approach and $\mathrm{FeCl}_{3}$-promoted Friedel-Crafts reaction. A highly soluble trithiocarbonate functionalized GO, which is the key $2 \mathrm{D}$ template in this approach, was used as a 2D micro-CTA for RAFT polymerization. The resulting GHCPs exhibited superhydrophobic behavior (contact angle $>150^{\circ}$ ) and a high specific surface area of up to $1224 \mathrm{~m}^{2} \mathrm{~g}^{-1}$. They also exhibited an enhanced $\mathrm{H}_{2}$ capacity (1.27 wt\%) at $77 \mathrm{~K}$ and $\mathrm{CO}_{2}$ capacity (9.9 wt\%) at $273 \mathrm{~K}$ at a relative pressure of $0.99 \mathrm{in}$ comparison with unadorned HCP without using the graphene template. The high specific surface areas and enhanced $\mathrm{H}_{2}$ and $\mathrm{CO}_{2}$ capacities might come from the contribution of the 2D graphene template and the 2D morphology. The GHCPs were also used to prepare porous carbon nanosheets without a template-removing operation. The as-prepared porous carbon nanosheets were successfully utilized in electrochemical capacitors, which makes this method a good approach for the preparation of $2 \mathrm{D}$ HCPs and $2 \mathrm{D}$ porous carbons with wide applications.

\section{Acknowledgements}

This work was financially supported by the National Basic Research Program of China (973 Program: 2013CBA01602 and 2012CB933404), the Natural Science Foundation of China 
(51403126), and Shanghai Jiao Tong University (211 and 985 Project). We also thank the Instrumental Analysis Center of Shanghai Jiao Tong University for providing some measurements.

\section{Notes and references}

1 D. Wu, F. Xu, B. Sun, R. Fu, H. He and K. Matyjaszewski, Chem. Rev., 2012, 112, 3959.

2 M. S. Silverstein, N. R. Cameron and M. A. Hillmyer, Porous Polymers, John Wiley \& Sons, 2011.

3 R. Dawson, A. I. Cooper and D. J. Adams, Prog. Polym. Sci., 2012, 37, 530 .

4 A. Thomas, Angew. Chem., Int. Ed., 2010, 49, 8328.

5 J. W. Colson and W. R. Dichtel, Nat. Chem., 2013, 5, 453.

6 X. Feng, X. S. Ding and D. L. Jiang, Chem. Soc. Rev., 2012, 41, 6010.

7 L. Chen, Y. Honsho, S. Seki and D. L. Jiang, J. Am. Chem. Soc., 2010, 132, 6742.

8 X. M. Liu, Y. H. Xu and D. L. Jiang, J. Am. Chem. Soc., 2012, 134, 8738 .

9 H. A. Patel, S. Hyun Je, J. Park, D. P. Chen, Y. Jung, C. T. Yavuz and A. Coskun, Nat. Commun., 2013, 4, 1357.

10 Y. Zhu, H. Long and W. Zhang, Chem. Mater., 2013, 25, 1630.

11 X. Wang, K. Maeda, A. Thomas, K. Takanabe, G. Xin, J. M. Carlsson, K. Domen and M. Antonietti, Nat. Mater., 2009, 8, 76.

12 R. Palkovits, M. Antonietti, P. Kuhn, A. Thomas and F. Schüth, Angew. Chem., Int. Ed., 2009, 48, 6909.

13 M. G. Schwab, B. Fassbender, H. W. Spiess, A. Thomas, X. Feng and K. Müllen, J. Am. Chem. Soc., 2009, 131, 7216.

14 Y. Kou, Y. Xu, Z. Guo and D. Jiang, Angew. Chem., Int. Ed., 2011, 50, 8753.

15 S. Xu, Y. Luo and B. Tan, Macromol. Rapid Commun., 2013, 34, 471.

16 M. Seo, S. Kim, J. Oh, S.-J. Kim and M. A. Hillmyer, J. Am. Chem. Soc., 2014, 137, 600.

17 F. S. Macintyre, D. C. Sherrington and L. Tetley, Macromolecules, 2006, 39, 5381.

18 L. Pan, Q. Chen, J.-H. Zhu, J. Yu, Y. He and B.-H. Han, Polym. Chem., 2015, 6, 2478.

19 J. Germain, J. M. J. Frechet and F. Svec, Chem. Commun., 2009, 1526.

20 J. Germain, J. Hradil, J. M. J. Frechet and F. Svec, Chem. Mater., 2006, 18, 4430.

21 A. P. Katsoulidis and M. G. Kanatzidis, Chem. Mater., 2011, 23, 1818.

22 X.-J. Chen, D. Ngoc Phuoc, J. Zhao, Y.-T. Wang, S.-P. Li and F. Svec, J. Sep. Sci., 2012, 35, 1502.

23 Y. Lv, Z. Lin and F. Svec, Anal. Chem., 2012, 84, 8457.

24 C. D. Wood, B. Tan, A. Trewin, F. Su, M. J. Rosseinsky, D. Bradshaw, Y. Sun, L. Zhou and A. I. Cooper, Adv. Mater., 2008, 20, 1916.

25 Y. Ouyang, H. Shi, R. Fu and D. Wu, Sci. Rep., 2013, 3, 1430 .
26 K. S. Novoselov, A. K. Geim, S. V. Morozov, D. Jiang, Y. Zhang, S. V. Dubonos, I. V. Grigorieva and A. A. Firsov, Science, 2004, 306, 666.

27 A. K. Geim and K. S. Novoselov, Nat. Mater., 2007, 6, 183.

28 X. Zhuang, F. Zhang, D. Wu, N. Forler, H. Liang, M. Wagner, D. Gehrig, M. R. Hansen, F. Laquai and X. Feng, Angew. Chem., Int. Ed., 2013, 52, 9668.

29 X. Zhuang, Y. Chen, L. Wang, K.-G. Neoh, E.-T. Kang and C. Wang, Polym. Chem., 2014, 5, 2010.

30 X. Zhuang, Y. Chen, G. Liu, P. P. Li, C. X. Zhu, E. T. Kang, K. G. Neoh, B. Zhang, J. H. Zhu and Y. X. Li, Adv. Mater., 2010, 22, 1731.

31 X. Zhuang, F. Zhang, D. Wu and X. Feng, Adv. Mater., 2014, 26, 3081.

32 C. A. Cao, X. Zhuang, Y. Su, Y. Zhang, F. Zhang, D. Wu and X. Feng, Polym. Chem., 2014, 5, 2057.

33 Y. Zhang, X. Zhuang, Y. Su, F. Zhang and X. Feng, J. Mater. Chem. A, 2014, 2, 7742.

34 X. Huang, X. Qi, F. Boey and H. Zhang, Chem. Soc. Rev., 2012, 41, 666.

35 S. Eigler and A. Hirsch, Angew. Chem., Int. Ed., 2014, 53, 7720 .

36 H. J. Salavagione, G. Martinez and G. Ellis, Macromol. Rapid Commun., 2011, 32, 1771.

37 M. Fang, K. Wang, H. Lu, Y. Yang and S. Nutt, J. Mater. Chem., 2009, 19, 7098.

38 S. H. Lee, D. R. Dreyer, J. An, A. Velamakanni, R. D. Piner, S. Park, Y. Zhu, S. O. Kim, C. W. Bielawski and R. S. Ruoff, Macromol. Rapid Commun., 2010, 31, 281.

39 H. M. Etmimi, M. P. Tonge and R. D. Sanderson, J. Polym. Sci., Part A: Polym. Chem., 2011, 49, 1621.

40 F. Beckert, C. Friedrich, R. Thomann and R. Mülhaupt, Macromolecules, 2012, 45, 7083.

41 B. Zhang, Y. Chen, L. Xu, L. Zeng, Y. He, E. T. Kang and J. Zhang, J. Polym. Sci., Part A: Polym. Chem., 2011, 49, 2043.

42 Y. Li, X. Li, C. Dong, Y. Li, P. Jin and J. Qi, Biosens. Bioelectron., 2009, 25, 306.

43 Y. Li, X. Li, C. Dong, J. Qi and X. Han, Carbon, 2010, 48, 3427.

44 Y. Yang, X. Song, L. Yuan, M. Li, J. Liu, R. Ji and H. Zhao, J. Polym. Sci., Part A: Polym. Chem., 2012, 50, 329.

45 Y. Yang, J. Wang, J. Zhang, J. Liu, X. Yang and H. Zhao, Langmuir, 2009, 25, 11808.

46 W. S. Hummers and R. E. Offeman, J. Am. Chem. Soc., 1958, 80, 1339.

47 J. T. Lai, D. Filla and R. Shea, Macromolecules, 2002, 35, 6754.

48 B. Zhang, J. Wang, Y. Chen, D. Fruchtl, B. Yu, X. D. Zhuang, N. He and W. J. Blau, J. Polym. Sci., Part A: Polym. Chem., 2010, 48, 3161.

49 J. H. Ahn, J. E. Jang, C. G. Oh, S. K. Ihm, J. Cortez and D. C. Sherrington, Macromolecules, 2006, 39, 627.

50 C. D. Wood, B. Tan, A. Trewin, H. Niu, D. Bradshaw, M. J. Rosseinsky, Y. Z. Khimyak, N. L. Campbell, R. Kirk and E. Stöckel, Chem. Mater., 2007, 19, 2034. 
51 S. Yang, X. Feng, L. Wang, K. Tang, J. Maier and K. Müllen, Angew. Chem., Int. Ed., 2010, 49, 4795.

52 B. Li, F. Su, H.-K. Luo, L. Liang and B. Tan, Microporous Mesoporous Mater., 2011, 138, 207.

53 X. Zhuang, Y. Mai, D. Wu, F. Zhang and X. Feng, Adv. Mater., 2015, 27, 403.

54 P.-G. Ren, D.-X. Yan, X. Ji, T. Chen and Z.-M. Li, Nanotechnology, 2011, 22, 055705.

55 N. N. Chai, J. Zeng, K. G. Zhou, Y. L. Xie, H. X. Wang, H. L. Zhang, C. Xu, J. X. Zhu and Q. Y. Yan, Chem. - Eur. J., 2013, 19, 5948.

56 M. Xue, F. Li, J. Zhu, H. Song, M. Zhang and T. Cao, Adv. Funct. Mater., 2012, 22, 1284.

57 K. K. Sadasivuni, A. Saiter, N. Gautier, S. Thomas and Y. Grohens, Colloid Polym. Sci., 2013, 291, 1729.

58 K. Haubner, J. Murawski, P. Olk, L. M. Eng, C. Ziegler, B. Adolphi and E. Jaehne, ChemPhysChem, 2010, 11, 2131.
59 B.-S. Ko, J.-Y. Sohn and J. Shin, Polymer, 2012, 53, 4652.

60 J. Rouquerol, F. Rouquerol and K. S. Sing, Absorption by Powders and Porous Solids, Academic Press, 1999.

61 R. Pierotti and J. Rouquerol, Pure Appl. Chem., 1985, 57, 603.

62 Y. Luo, S. Zhang, Y. Ma, W. Wang and B. Tan, Polym. Chem., 2013, 4, 1126.

63 Z. Wang, S. W. Yuan, A. Mason, B. Reprogle, D. J. Liu and L. P. Yu, Macromolecules, 2012, 45, 7413.

64 W. G. Lu, D. Q. Yuan, D. Zhao, C. I. Schilling, O. Plietzsch, T. Muller, S. Brase, J. Guenther, J. Blumel, R. Krishna, Z. Li and H. C. Zhou, Chem. Mater., 2010, 22, 5964.

65 Y. Zhao, X. Liu, K. X. Yao, L. Zhao and Y. Han, Chem. Mater., 2012, 24, 4725.

66 M. G. Rabbani and H. M. El-Kaderi, Chem. Mater., 2012, 24, 1511. 